\title{
Prefilter-Based Reconfigurable Reconstructor for Time-Interleaved ADCs With Missing Samples
}

Anu Kalidas Muralidharan Pillai and Håkan Johansson

\author{
Linköping University Post Print
}

\section{Tweet}

N.B.: When citing this work, cite the original article.

Anu Kalidas Muralidharan Pillai and Håkan Johansson, Prefilter-Based Reconfigurable Reconstructor for Time-Interleaved ADCs With Missing Samples, 2015, IEEE Transactions on Circuits and Systems - II - Express Briefs, (62), 4, 392-396.

http://dx.doi.org/10.1109/TCSII.2014.2387651

(C)2015 IEEE. Personal use of this material is permitted. However, permission to reprint/republish this material for advertising or promotional purposes or for creating new collective works for resale or redistribution to servers or lists, or to reuse any copyrighted component of this work in other works must be obtained from the IEEE.

\section{http://ieeexplore.ieee.org/}

Postprint available at: Linköping University Electronic Press

http://urn.kb.se/resolve?urn=urn:nbn:se:liu:diva-117769 


\title{
Prefilter-Based Reconfigurable Reconstructor for Time-Interleaved ADCs with Missing Samples
}

\author{
Anu Kalidas M. Pillai, Student Member, IEEE, and Håkan Johansson, Senior Member, IEEE
}

\begin{abstract}
This brief proposes a reconstruction scheme for the compensation of frequency-response mismatch errors at the output of a time-interleaved analog-to-digital converter (TI-ADC) with missing samples. The missing samples are due to sampling instants reserved for estimating the channel mismatch errors in the TI-ADC. Compared to previous solutions, the proposed scheme offers a substantially lower computational complexity.
\end{abstract}

Index Terms-Reconstruction, frequency-response mismatch errors, time-interleaved analog-to-digital converters, reconfigurable reconstructors.

\section{INTRODUCTION}

Frequency-response mismatches between the channel converters in a time-interleaved analog-to-digital converter (TIADC) degrade the resolution. A common technique to improve the resolution is through digital calibration at the output of the TI-ADC. In this technique, the mismatch parameters are estimated from the TI-ADC output which are then used to reconstruct the output so as to compensate for the mismatch errors. In [1], an iterative background estimation scheme that achieves robust estimation compared to other background estimation schemes was introduced. In this scheme, a known calibration signal is injected at predefined sampling instants. The mismatch parameters are then estimated by using the TIADC output samples corresponding to the calibration signal. Since the input signal is not sampled at the sampling instants reserved for the calibration signal, the sampled signal at the output of the TI-ADC will contain missing samples.

In this brief, we focus on reducing the complexity of the reconstructor. Hence, we assume that the mismatch parameters are estimated and available. In [1], an iterative reconstruction scheme was proposed which recovers the missing samples and provides compensation for the mismatch errors. However, [1] makes use of recursive structures which, in addition to limiting the maximal data rate, can lead to stability problems. Moreover, for this scheme, the computational complexity, measured in terms of the number of multiplications required per corrected output sample, is very high. A sub-band based reconstruction scheme that utilizes nonrecursive structures, with a lower overall computational complexity was proposed in [2]. A constrained time-varying finite-length impulse response (FIR) reconstructor was proposed in [3] and achieved even lower computational complexity compared to the scheme in [2] at the cost of increased online redesign complexity. It should

The authors are with the Division of Communication Systems, Department of Electrical Engineering, Linköping University, Linköping, Sweden (email: kalidas@isy.liu.se, hakanj@isy.liu.se).

Copyright (c) 2014 IEEE. Personal use of this material is permitted However, permission to use this material for any other purposes must be obtained from the IEEE by sending an email to pubs-permissions@ieee.org be noted that [2] and [3] assumed that the TI-ADC suffers only from static time-skew errors. However, for high operating frequencies and/or resolution, the reconstructor should also compensate for frequency-dependent mismatch errors between the channel ADCs [4].

In Section III of this brief, we propose a reconfigurable reconstruction scheme that compensates for both the static and frequency-dependent mismatch errors in the presence of missing samples. The proposed scheme uses a nonrecursive structure consisting of a number of fixed subfilters and variable multipliers. Whenever the mismatch parameters change, the reconstructor can be reconfigured directly by updating the variable multipliers with the values obtained from the estimation block. Thus, unlike the reconstruction schemes in [2] and [3], the proposed scheme requires no online redesign block. Also, as illustrated using design examples in Section IV, compared to the reconstruction scheme in [1] which requires no online redesign, the computational complexity of the proposed scheme is substantially lower. Finally, it is noted that the reconstruction of missing samples from bandlimited signals has been considered in other contexts [5]. However, they do not address the problem considered in this brief where additional mismatch errors are also present.

\section{BACKGRoUnd AND PREREQUisites}

Assume that a continuous-time input signal $x_{\mathrm{a}}(t)$ that is bandlimited to $\omega_{\mathrm{c}}<\pi / T$, is sampled by an $M$-channel TI-ADC where the channel ADCs operate at a sampling frequency of $1 /(M T)$. If all the $M$ channels are perfectly matched with each other, we obtain a uniformly sampled signal $x(n)$. However, in practice, the channel frequency responses $Q_{n}(j \omega T), n=0,1, \ldots, M-1$, are not perfectly matched resulting in an $M$-periodically nonuniformly sampled signal $v(n)$ at the output of the TI-ADC. For example, if the TI-ADC only suffers from static time-skew errors, $Q_{n}(j \omega T)=e^{j \omega T \varepsilon_{n}}$ where $\varepsilon_{n}$ represents the difference between the actual and the desired time skew for the sampling clock in the $n$th channel resulting in $v(n)=x_{\mathrm{a}}\left(n T+\varepsilon_{n} T\right)$. The TI-ADC output $v(n)$ can be considered as obtained by sampling the output of a time-varying continuous-time system such that [6]

$$
v(n)=\frac{1}{2 \pi} \int_{-\omega_{\mathrm{c}} T}^{\omega_{\mathrm{c} T}} Q_{n}(j \omega T) X\left(e^{j \omega T}\right) e^{j \omega T n} \mathrm{~d}(\omega T)
$$

where $Q_{n}(j \omega T)=Q_{n+M}(j \omega T), \forall n \in \mathbb{Z}$ and $X\left(e^{j \omega T}\right)$ represents the Fourier transform of $x(n)$.

A digital calibration block reconstructs the uniform-grid samples $x(n)$ from the sampled signal $v(n)$. The calibration involves estimation of the mismatch parameters from $v(n)$ 
and compensating for the frequency-response mismatch errors using the estimates. A background estimation technique was proposed in [1], where some of the sampling instants of the TIADC were reserved for estimating the mismatch parameters. It was shown that compared to blind estimation schemes, the scheme in [1] enables robust estimation. In this scheme, a known calibration signal is applied to the input of the TIADC at predefined sampling instants $t=r M_{c} T, \forall r \in \mathbb{Z}$. The resulting nonuniformly sampled calibration signal is then compared with a known reference sequence to estimate the mismatch parameters. As the estimation requires samples from all the channel ADCs, $M_{c}$ is chosen such that $M$ and $M_{c}$ are co-prime. In a TI-ADC utilizing the above estimation scheme, the input to the reconstructor, $y(n)$, contains missing inputsignal samples and is given by

$$
y(n)= \begin{cases}0, & n=r M_{c} \\ v(n), & n \neq r M_{c} .\end{cases}
$$

Using (1), we can rewrite (2) as

$$
y(n)=\frac{1}{2 \pi} \int_{-\omega_{\mathrm{c}} T}^{\omega_{\mathrm{c}} T} \bar{Q}_{n}(j \omega T) X\left(e^{j \omega T}\right) e^{j \omega T n} \mathrm{~d}(\omega T)
$$

where

$$
\bar{Q}_{n}(j \omega T)= \begin{cases}0, & \forall n=r M_{c} \\ Q_{n \bmod M}(j \omega T), & \forall n \neq r M_{c}\end{cases}
$$

with $\bar{Q}_{n}(j \omega T)=\bar{Q}_{n+M M_{c}}(j \omega T)$. As can be seen from (3) and (4), due to the missing samples, $y(n)$ can be considered as an $N$-periodically nonuniformly sampled version of the input with $N=M M_{c}$. Hence, as proposed in [3], the reconstruction can be performed using a constrained timevarying FIR reconstructor consisting of $N$ separate FIR filters $c_{n}(k), n=0,1, \ldots, N-1$, with some of the coefficients in $c_{n}(k)$ being zero-valued. In such a reconstructor, the difference between $A_{n}(j \omega T)$ and unity determine how well the reconstructed signal $\tilde{x}(n)$ approximates the ideal uniformgrid samples $x(n)$. Here,

$$
A_{n}(j \omega T)=\sum_{i=1}^{R_{n}} c_{n}\left(k_{i}\right) \bar{Q}_{n-k_{i}}(j \omega T) e^{-j \omega T k_{i}}
$$

where $R_{n}, n=0,1, \ldots, N-1$, represent the number of non-zero impulse response coefficients in $c_{n}(k)$ and $k_{i}$, $i=1,2, \ldots, R_{n}$, are the indices of the non-zero coefficients. For a given reconstruction problem, the constrained timevarying reconstructor gives the minimal order for the overall reconstructor, but it requires online redesign whenever the channel frequency-response mismatch errors change. This online redesign significantly increases the total implementation complexity as it involves $N$ separate matrix inversions [3]. Moreover, since the filter coefficients are redetermined online, all the multipliers in the constrained time-varying FIR reconstructor need to be variable-coefficient multipliers which are expensive compared to fixed-coefficient multipliers.

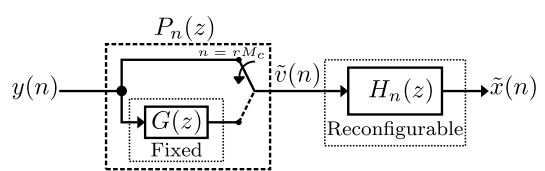

Fig. 1. Proposed reconstruction scheme.

\section{Proposed Reconstructor}

In the proposed reconstruction scheme shown in Fig. 1, the overall complexity of the reconstructor is reduced by using a constrained two-mode time-varying FIR prefilter $P_{n}(z)$ to approximately recover the missing samples in $y(n)$. After this, the reconstructed output $\tilde{x}(n)$ is obtained by using a timevarying filter $H_{n}(z)$ which compensates for the remaining errors.

\section{A. Two-Mode Time-Varying Prefilter}

Since the prefilter $P_{n}(z)$ only approximately recovers the missing samples in $y(n)$, its impulse response $p_{n}(k)$, composed of two impulse responses (two modes), is given by

$$
p_{n}(k)= \begin{cases}g(k), & \forall n=r M_{c} \\ \delta(k), & \forall n \neq r M_{c}\end{cases}
$$

where $r \in \mathbb{Z}, \delta(k)$ represents an impulse, and $g(k)$ is the impulse response of a linear-phase FIR filter. As can be seen from Fig. 1, the missing samples in $y(n)$, corresponding to $n=r M_{c}$, are approximately recovered using the fixed filter $G(z)$ whereas all the other samples in $y(n)$ are directly passed to the output $\tilde{v}(n)$. The missing samples in $y(n)$ restrict some of the impulse response coefficients in $g(k)$ to be zero. That is,

$$
g(k)=0, \quad \forall k=r M_{c} .
$$

Like in [3], the missing sample value in $y(n)$ at time index $n=$ $r M_{c}$ is perfectly recovered if $\sum_{i=1}^{R} g\left(k_{i}\right) \bar{Q}_{n-k_{i}}(j \omega T) e^{-j \omega T k_{i}}$ equals unity. Here, $R$ represents the number of non-zero coefficients in $g(k)$ and $k_{i}, i=1,2, \ldots, R$, are the indices of the non-zero impulse response coefficients. However, in order to approximately recover the missing samples, it suffices to determine the $R$ non-zero coefficients of $g(k)$ such that, $\sum_{i=1}^{R} g\left(k_{i}\right) e^{-j \omega T k_{i}}, k_{i} \in\left[-N_{G} / 2, \ldots, N_{G} / 2\right]$, approximates unity in the passband $\omega T \in\left[-\omega_{\mathrm{c}} T, \omega_{\mathrm{c}} T\right]$ with a certain tolerance. Here, $N_{G}$, the filter order of $g(k)$, is selected to be even so that the filter coefficients are symmetric.

\section{B. Reconfigurable Part}

It can be seen from Fig. 1 that the recovered sample value in $\tilde{v}(n)$ can be expressed for all $n=r M_{c}, r \in \mathbb{Z}$ as

$$
\tilde{v}(n)=y(n) * g(n)=\sum_{i=1}^{R} g\left(k_{i}\right) y\left(n-k_{i}\right) .
$$

In (8), $k_{i}, i=1,2, \ldots, R$, denote the indices of the non-zero impulse response coefficients. Using (1) and (2) in (8), we get

$$
\tilde{v}(n)=\frac{1}{2 \pi} \int_{-\omega_{\mathrm{c}} T}^{\omega_{\mathrm{c} T}} F_{n}(j \omega T) X\left(e^{j \omega T}\right) e^{j \omega T n} \mathrm{~d}(\omega T)
$$


where

$$
F_{n}(j \omega T)=\sum_{k=-N_{G} / 2}^{N_{G} / 2} g(k) Q_{n-k}(j \omega T) e^{-j \omega T k}
$$

with $g(k)$ constrained as in (7). In order to obtain a reconfigurable reconstructor, we approximate the channel frequency responses $Q_{n}(j \omega T), n=0,1, \ldots, M-1$, with $P$ th-order polynomials in $j \omega T[6]$ according to ${ }^{1}$

$$
Q_{n}(j \omega T) \approx 1+\sum_{q=1}^{P} d_{n}^{(q)}(j \omega T)^{q}
$$

The polynomials in (11) are determined by estimating the mismatch parameters from $v(n)$. The reconstruction problem is thus to recover $x(n)$ from $\tilde{v}(n)$ given the estimates for $d_{n}^{(q)}, n=0,1, \ldots, M-1, q=1,2, \ldots, P$. Using $(11)$ in (10) followed by some algebraic manipulation, we get $F_{n}(j \omega T)=1+\bar{F}_{n}(j \omega T)$ with

$\bar{F}_{n}(j \omega T)=\Delta G\left(e^{j \omega T}\right)+\sum_{q=1}^{P} \sum_{k=-N_{G} / 2}^{N_{G} / 2}(j \omega T)^{q} d_{n-k}^{(q)} g(k) e^{-j \omega T k}$

and $\Delta G\left(e^{j \omega T}\right)=G\left(e^{j \omega T}\right)-1$, represents the deviation of the frequency response of $g(k)$ from unity, in the passband. Substituting the expression for $F_{n}(j \omega T)$ in (9), the recovered sampled value in $\tilde{v}(n), n=r M_{c}$, can be expressed as

$$
\tilde{v}(n)=x(n)+\frac{1}{2 \pi} \int_{-\omega_{\mathrm{c}} T}^{\omega_{\mathrm{c}} T} \bar{F}_{n}(j \omega T) X\left(e^{j \omega T}\right) e^{j \omega T n} \mathrm{~d}(\omega T)
$$

where $x(n)$ is the uniform-grid sample value. Using (6), we can extend (13) to represent the output of $p_{n}(k), \forall n \in \mathbb{Z}$, as

$$
\tilde{v}(n)=x(n)+\tilde{e}(n)
$$

where $\tilde{e}(n)$ represents the remaining errors at the output of the prefilter and is given by

$$
\tilde{e}(n)=\frac{1}{2 \pi} \int_{-\omega_{\mathrm{c}} T}^{\omega_{\mathrm{c}} T} E_{n}(j \omega T) X\left(e^{j \omega T}\right) e^{j \omega T n} \mathrm{~d}(\omega T) .
$$

In $(15)$,

$$
\begin{aligned}
E_{n}(j \omega T)= & \Delta P_{n}\left(e^{j \omega T}\right) \\
& +\sum_{q=1}^{P} \sum_{k=-N_{G} / 2}^{N_{G} / 2}(j \omega T)^{q} d_{n-k}^{(q)} p_{n}(k) e^{-j \omega T k}
\end{aligned}
$$

with

$$
\Delta P_{n}\left(e^{j \omega T}\right)= \begin{cases}\Delta G\left(e^{j \omega T}\right), & \forall n=r M_{c} \\ 0, & \forall n \neq r M_{c} .\end{cases}
$$

It can be seen from (16) and (6) that the remaining errors in $\tilde{v}(n)$ come from three components namely the channel frequency-response mismatch errors, the approximation error in the frequency response of the prefilter, and the frequencyresponse mismatch errors modified by the prefilter.

\footnotetext{
${ }^{1}$ In (11), we have assumed that there are no static gain mismatches as they can be easily compensated for by multiplying $y(n)$ by a single time-varying multiplier. Such a multiplier only increases the computational complexity $\mathcal{C}_{\mathrm{m}}$ in (18) by one.
}

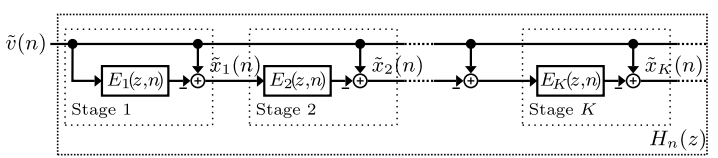

Fig. 2. Realization of $H_{n}(z)$ using an iterative structure.

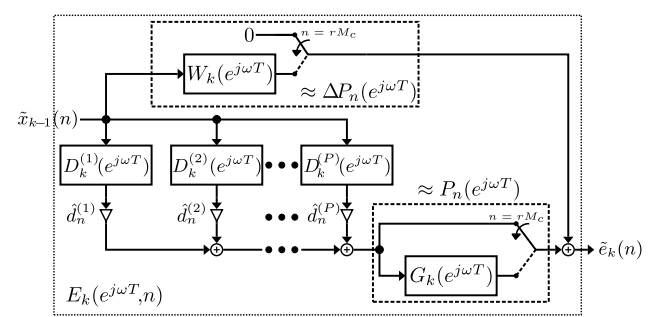

Fig. 3. Realization of $E_{k}\left(e^{j \omega T}, n\right)$ in the $k$ th stage of $H_{n}(z)$.

Expressing the prefilter output as in (14) allows us to realize the $H_{n}(z)$ using the iterative compensation structure shown in Fig. 2. If there were no missing samples, each time-varying filter $E_{k}(z, n), k \in[1,2, \ldots, K]$, in Fig. 2 can be realized using a Farrow structure without the input signal branch, like in [7]. However, here, due to the missing samples and the prefilter, each $E_{k}(z, n)$ is realized using a Farrow structure with additional blocks, $W_{k}\left(e^{j \omega T}\right)$ and $G_{k}\left(e^{j \omega T}\right)$, as shown in Fig. 3. The linear-phase FIR subfilters $D_{k}^{(q)}\left(e^{j \omega T}\right), G_{k}\left(e^{j \omega T}\right)$, and $W_{k}\left(e^{j \omega T}\right), q \in[1,2, \ldots, P], k \in[1,2, \ldots, K]$, approximate $(j \omega T)^{q}, G\left(e^{j \omega T}\right)$, and $G\left(e^{j \omega T}\right)-1$, respectively, in the passband $\omega T \in\left[-\omega_{\mathrm{c}} T, \omega_{\mathrm{c}} T\right]$. The impulse response coefficients of $G\left(e^{j \omega T}\right), G_{k}\left(e^{j \omega T}\right), W_{k}\left(e^{j \omega T}\right)$, and $D_{k}^{(q)}\left(e^{j \omega T}\right)$, $q=1,2, \ldots, P, k=1,2, \ldots, K$, are determined offline and then fixed. Whenever the frequency-response mismatch errors change, the reconstructor can be reconfigured directly by updating the variable multipliers $\hat{d}_{n}^{(q)}, q=1,2, \ldots, P$, $n=0,1, \ldots, M-1$, with the set of coefficients obtained from the estimation block.

\section{Complexity}

As the coefficients of the subfilters are determined offline and fixed, they can be implemented using fixed-coefficient multipliers. Moreover, as we use linear-phase FIR subfilters, the number of such fixed-coefficient multipliers is around half their filter order. Further, as some taps in the prefilter are equal to zero, the number of fixed-coefficient multipliers required for the prefilter is even less than $N_{G} / 2$. Thus, the computational complexity of the proposed reconstructor, measured in terms of the number of multiplications required per corrected output sample, is given by ${ }^{2}$

$$
\mathcal{C}_{\mathrm{m}}=\frac{R}{2 M_{c}}+\sum_{k=1}^{K}\left(\frac{R_{k}}{2 M_{c}}+P_{\max , k}+\sum_{q=1}^{P_{\max , k}} \mathcal{C}_{D_{k}^{(q)}}\right)
$$

where $R$ and $R_{k}$ represent the number of non-zero coefficients in $G\left(e^{j \omega T}\right)$ and $G_{k}\left(e^{j \omega T}\right)$, respectively, $P_{\max , k} \in[1,2, \ldots, P]$

${ }^{2}$ In (18), it is assumed that the reconstructor compensates samples from all the channels. In practice, the value for $\mathcal{C}_{\mathrm{m}}$ will be lower than that obtained from (18) as one of the channels, which is assumed to be the reference channel, requires no compensation. 
represents the maximum order of the differentiator in the $k$ th stage, $\mathcal{C}_{D_{k}^{(q)}}=N_{D_{k}^{(q)}} / 2+1-(q \bmod 2)$ is the number of multiplications required for the filter $D_{k}^{(q)}(z)$ whose filter order is $N_{D_{k}^{(q)}}$. Since, during reconfiguration, the multipliers $\hat{d}_{n}^{(q)}, q=1,2, \ldots, P$, in each stage are updated with the corresponding newly estimated values, they are implemented using general multipliers.

\section{Design}

In the design, we use a distortion function $V_{0}\left(e^{j \omega T}\right)$ and $N-1$ aliasing functions $V_{m}\left(e^{j \omega T}\right), m=1,2, \ldots, N-1$, $N=M M_{c}$, to obtain a measure of the overall reconstruction error. Here, each $V_{m}\left(e^{j \omega T}\right), m \in[0,1, \ldots, N-1]$, is obtained through a linear combination of the functions $A_{n}(j \omega T)$, $n=0,1, \ldots, N-1$, as given by (22) in [6]. For perfect reconstruction (PR), $V_{0}\left(e^{j \omega T}\right)$ should be equal to unity whereas $V_{m}\left(e^{j \omega T}\right), m=1,2, \ldots, N-1$, should be equal to zero. In practice, however, we require only approximate $\mathrm{PR}$ and the reconstruction error depends on how closely the distortion and the aliasing functions approximate unity and zero, respectively, in the passband region $\left[-\omega_{\mathrm{c}} T, \omega_{\mathrm{c}} T\right][6]$. Thus, given the values for $P, K$, and the orders of the subfilters, the design of the overall reconstructor involves determining the coefficients of the subfilters such that for a maximum specified reconstruction error $\delta$,

$$
\left|V_{m}\left(e^{j \omega T}\right)-a_{m}\right| \leq \delta, \omega T \in\left[-\omega_{\mathrm{c}} T+\frac{2 \pi m}{M}, \omega_{\mathrm{c}} T+\frac{2 \pi m}{M}\right]
$$

for $m=0,1, \ldots, N-1$. In (19), $a_{0}=1$ and $a_{m}=0$ for $m=1,2, \ldots, N-1$. Here, we propose to determine the coefficients of the subfilters separately as the cascaded filter structure makes their joint optimization cumbersome.

It is noted that the filter $W_{k}(z)$ in each stage is only required to compensate for the error $\Delta G\left(e^{j \omega T}\right)$ introduced by the prefilter $G(z)$. However, based on experiments, it is observed that the overall computational complexity is lower if the error $\Delta G\left(e^{j \omega T}\right)$ introduced by the prefilter is equal to the desired reconstruction error, thereby eliminating the need for the $W_{k}(z)$ filter in each stage. Thus, here, we assume that $N_{G}$ is selected such that $W_{k}(z)$ is not required.

For a given set of $\omega_{\mathrm{c}} T, P, \delta$, and the specified range of values taken by the mismatch parameters $\hat{d}_{n}^{(q)}, q=1,2, \ldots, P$, $n=0,1, \ldots, M-1$, the value of $K$ and the filter orders $N_{G}$, $N_{G_{k}}$, and $N_{D_{k}^{(p)}}, p=1,2, \ldots, P, k=1,2, \ldots, K$, are determined using the steps outline below. Here, the overall error is computed by taking the maximum of the reconstruction errors evaluated for a number of different combinations of uniformly distributed mismatch parameters within the specified range.

1) In order to determine the value of $K$, the overall error is determined for each value of $K$, starting with $K=1$, until the specified reconstruction error is achieved. Here, all the subfilters are overdesigned with filter approximation errors equal to $\delta$.

2) To determine the value of $N_{G}$, we use an $H_{n}(z)$ consisting of $K$ (determined in Step 1) stages with overdesigned differentiators and $G_{k}(z), k=1,2, \ldots, K$, with filter orders $N_{G_{k}}=N_{G}$. Starting with the value of $N_{G}$ for which the approximation error in the prefilter is equal to $\delta$, the minimal value for $N_{G}$ is determined by decreasing $N_{G}$ until the overall error is equal to or slightly lower than the specified reconstruction error.

3) After $N_{G}$ is determined and fixed, we follow the same procedure as in Step 2 to determine $N_{G_{k}}, k \in$ $[1,2, \ldots, K]$, but with $N_{G}$ as the starting value for $N_{G_{k}}$.

4) In this step, to determine the filter order of the differentiators, we use the values for $K, N_{G}$, and $N_{G_{k}}$, $k=1,2, \ldots, K$, determined in Steps $1-3$, respectively. The initial value for each $N_{D_{k}^{(p)}}, p \in[1,2, \ldots, P]$, $k \in[1,2, \ldots, K]$, is set such that the corresponding $p$ thorder differentiator $D_{k}^{(p)}$ has an approximation error less than $\delta$. Next, the filter order of the differentiators in each stage is determined one-by-one. Starting with $D_{1}^{(1)}$ and keeping the filter order of all the other differentiators unchanged, we decrease the value of $N_{D_{1}^{(1)}}$ from its initial value until the overall error becomes $\delta$. Then, we replace the initial value of $N_{D_{1}^{(1)}}$ with the newly determined value and repeat the same procedure to determine $N_{D_{1}^{(2)}}$ and so on, up to $N_{D_{K}^{(P)}}$.

5) From different combinations of $N_{D_{k}^{(p)}}, p=1,2, \ldots, P$, $k=1,2, \ldots, K$, around the values determined in Step 4 , select the combination that satisfies the reconstruction error requirement and has the lowest $\mathcal{C}_{\mathrm{m}}$. If any $N_{D_{k}^{(p)}}$ is equal to zero, check whether the corresponding $D_{k}^{(p)}(z)$ can be removed from the implementation without affecting the reconstruction error requirement.

\section{DESIGN EXAMPLES}

Example 1: The purpose of this example is to compare the computational complexity of the proposed method with that of the existing methods. We also show that by increasing the number of stages $K$ and the order of the subfilters, any arbitrarily low reconstruction error can be achieved provided $P$ is chosen such that $Q_{n}(j \omega T)$ are accurately modeled. For comparison, we use the four-channel TI-ADC $(M=4)$ case considered in Example A in Section VI of [1]. Thus, it is assumed that the timing mismatches in the channel ADCs are $\varepsilon_{0,1,2,3}=[0.01,-0.05,0.04,-0.03]$, with every seventh sample being used by the estimator $\left(M_{c}=7\right)$ and the bandwidth of the reconstructor, $\omega_{\mathrm{c}} T=0.8 \pi$. Further, it is required that, after reconstruction, the aliasing terms at the output should be below $-50 \mathrm{~dB}$.

Since the TI-ADC here is assumed to have only timing mismatches, the channel frequency responses are given by $Q_{n}(j \omega T)=e^{j \omega T \varepsilon_{n}}, n=0,1,2,3$. To be consistent with the corresponding examples in [1]-[3], here, we assume that the reconstructor compensates samples from all the channels without using any channel as the reference channel. Thus, using (11) and $P=2$ and assuming that the estimation block estimates the mismatch parameters sufficiently accurately, we get $\hat{d}_{n}^{(1)}=\varepsilon_{n}$ and $\hat{d}_{n}^{(2)}=\varepsilon_{n}^{2} / 2$. To keep the aliasing terms below $-50 \mathrm{~dB}$ for any $\varepsilon_{n} \in[-0.05,0.05]$, the proposed reconstructor needs $K=2$ stages and a prefilter of order 


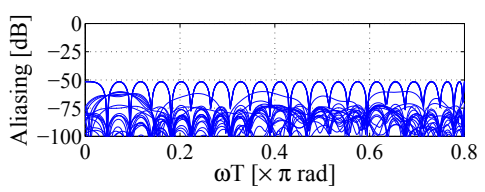

Fig. 4. Example 1: Magnitude of the aliasing functions $V_{m}\left(e^{j \omega T}\right), m=$ $1,2, \ldots, 27$ for $\varepsilon_{0,1,2,3}=[0.01,-0.05,0.04,-0.03]$ and $K=2$.

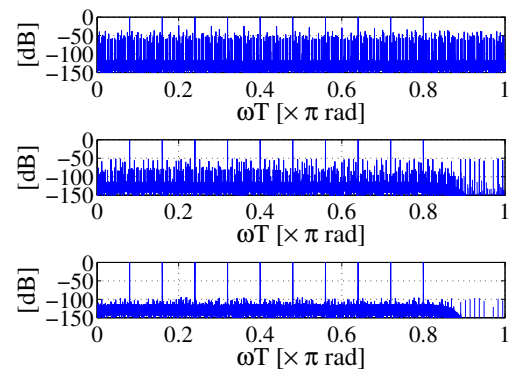

Fig. 5. Example 1: Spectrum of the uncompensated TI-ADC output with missing samples, $y(n)$ (top), the reconstructed sequence, $\tilde{x}(n)$, with $K=2$ (middle) and with $K=3$ (bottom). The input is a multi-tone sinusoidal signal with tones at $\omega=2 \pi r /(25 T), r=1,2, \ldots, 10$, and is quantized to 16 bits.

$N_{G}=48$ with $R=42$ non-zero impulse response coefficients. Further, $N_{G_{1,2}}=[34,48], N_{D_{1}^{(1)}}=12$, and $N_{D_{2}^{(1)}}=[12,10]$. As shown in Fig. 4, for the given time-skew errors, the maximum amplitude of all the aliasing terms $V_{m}\left(e^{j \omega T}\right)$, $m=1,2, \ldots, 27$, is below $-50 \mathrm{~dB}$. It can be seen from Table I that the proposed reconstructor has significantly lower overall complexity (including control complexity) compared to the existing reconstruction schemes. Further, for the given $\varepsilon_{0,1,2,3}$ combination, the proposed reconstructor can reduce the magnitude of the aliasing terms to below $-94 \mathrm{~dB}$ using $P=3, K=3, N_{G}=102(R=88), N_{G_{1,2,3}}=[34,88,102]$, $N_{D_{1}^{(1)}}=8, N_{D_{2}^{(1,2)}}=[20,4]$, and $N_{D_{3}^{(1,2,3)}}=[30,10,8]$, with a computational complexity $\mathcal{C}_{\mathrm{m}}=68$. On the other hand, the reconstructor in [1] requires around 1100 multiplications per corrected output sample to attain a similar performance. Also, reconfiguring the constrained time-varying reconstructor in [3] for such low reconstruction errors is impractical as it requires inverting large matrices online. Figure 5 shows the spectrum before and after reconstruction using the proposed reconstructor when a multi-tone signal with frequencies as in [1]-[3] is applied at the input of the TI-ADC. The corresponding plots for the existing methods can be seen in Figs. 9(c) and (d) in [1], Fig. 4 in [2], and Fig. 3 in [3].

Example 2: In this example, we consider a TI-ADC with $M=4, M_{c}=7, \omega_{\mathrm{c}} T=0.8 \pi$, and with channel frequency responses $Q_{n}(j \omega T)=e^{j \omega T \varepsilon_{n}} /\left(1+j \omega T\left(1+r_{n}\right)\right), n=$ $0,1,2,3$, where $r_{n}=\left(1+\Delta_{n}\right) / 4 \pi$ and the parameters $\varepsilon_{n}$ and $\Delta_{n}$ correspond to the analog matching errors [6]. Like in [6], we assume that $\varepsilon_{n} \in[-0.02,0.02]$ and $\Delta_{n} \in[-0.005,0.005]$ and that the reconstruction is performed with the zeroth channel as the reference channel. In order to keep the aliasing terms below $-100 \mathrm{~dB}$, we require $P=4, K=4, N_{G}=108$, $N_{G_{1,2,3,4}}=[20,68,100,108], N_{D_{1}^{(1)}}=10, N_{D_{2}^{(1,2)}}=[14,2]$, $N_{D_{3}^{(1,2,3)}}=[18,4,6], N_{D_{4}^{(1,2,3,4)}} \stackrel{D_{1}}{=}[24,10,10,2]$, with $\mathcal{C}_{\mathrm{m}}=$

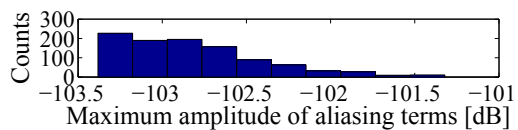

Fig. 6. Example 2: Histogram of the maximum amplitude of the aliasing terms for 1000 combinations of uniformly distributed $\varepsilon_{n}$ and $\Delta_{n}, n=$ $0,1,2,3$, within the range $\varepsilon_{n} \in[-0.02,0.02]$ and $\Delta_{n} \in[-0.005,0.005]$.

TABLE I

EXAMPLE 1: COMPLEXITY COMPARISON FOR $\delta=0.0032(-50 \mathrm{DB})$.

\begin{tabular}{|c|c|c|c|c|c|c|}
\hline & Order & \multicolumn{4}{|c|}{ Complexity $^{3}$} & Structure \\
& & $\mathcal{C}_{\mathrm{f}}$ & $\mathcal{C}_{\mathrm{v}}$ & $\mathcal{C}_{\mathrm{m}}$ & Redesign & \\
\hline \hline$[1]$ & 396 & 543 & 11 & 554 & None & Recursive \\
\hline$[2]$ & 960 & 27 & 48 & 75 & 1 & Nonrecursive \\
\hline$[3]$ & 68 & 0 & 31 & 31 & 28 & Nonrecursive \\
\hline Proposed & 154 & 28 & 3 & 31 & None & Nonrecursive \\
\hline
\end{tabular}

73. Figure 6 shows the histogram of the maximum amplitude of all the aliasing terms after reconstruction for uniformly distributed analog matching errors within the specified range. It can be seen from Fig. 6 that the aliasing terms are below $-100 \mathrm{~dB}$ for all the combinations of $\varepsilon_{n}$ and $\Delta_{n}$. It is also noted that, to keep the aliasing terms below $-50 \mathrm{~dB}$, the proposed reconstruction scheme requires a $\mathcal{C}_{\mathrm{m}}$ of only 17 .

\section{CONCLUSion}

This brief proposed a reconfigurable reconstruction scheme for TI-ADCs with missing samples. Compared to existing schemes, the proposed scheme achieved lower implementation complexity by using a prefilter to approximately recover the missing sample before compensating for the mismatch errors. The compensation filter was realized using an iterative structure consisting of a number of fixed subfilters and variable multipliers. We showed that the proposed scheme requires no online redesign as the reconstructor can be reconfigured by directly updating the variable multipliers.

\section{REFERENCES}

[1] K. M. Tsui and S. C. Chan, "A novel iterative structure for online calibration of $M$-channel time-interleaved ADCs," IEEE Trans. Instrum. Meas., vol. 63, no. 2, pp. 312-325, Feb. 2014.

[2] A. K. M. Pillai and H. Johansson, "A sub-band based reconstructor for $M$-channel time-interleaved ADCs with missing samples," in Proc. IEEE Int. Conf. Acoustics, Speech Signal Process., Florence, Italy, May 4-9, 2014, pp. 4126-4130.

[3] — "Two reconstructors for $M$-channel time-interleaved ADCs with missing samples," in Proc. IEEE Int. New Circuits and Systems Conf., Trois-Rivières, Canada, Jun. 22-25, 2014.

[4] H. Johansson and P. Löwenborg, "A least-squares filter design technique for the compensation of frequency response mismatch errors in timeinterleaved A/D converters," IEEE Trans. Circuits Syst. II, vol. 55, no. 11, pp. 1154-1158, Nov. 2008.

[5] F. Marvasti, Ed., Nonuniform Sampling: Theory and Practice. Kluwer Academic, Newyork, NY, USA, 2001.

[6] H. Johansson, "A polynomial-based time-varying filter structure for the compensation of frequency-response mismatch errors in time-interleaved ADCs," IEEE J. Sel. Topics Signal Process., vol. 3, no. 3, pp. 384-396, Jun. 2009.

[7] C. Vogel and S. Mendel, "A flexible and scalable structure to compensate frequency response mismatches in time-interleaved ADCs," IEEE Trans. Circuits Syst. I, vol. 56, no. 11, pp. 2463-2475, Nov. 2009.

${ }^{3} \mathcal{C}_{\mathrm{f}}$ and $\mathcal{C}_{\mathrm{v}}$ represent the number of fixed-coefficient and variable-coefficient multiplications per corrected output sample, respectively.

${ }^{4}$ Number of online matrix inversions. 